\title{
Avaliação dos distúrbios do controle sensório-motor em pessoas com dor cervical mecânica: uma revisão
}

\author{
Assessment of sensorimotor disturbance in mechanical neck pain: a review
}

\author{
Felipe José Jandre dos Reis ${ }^{[a]}$, Bruna Mafra ${ }^{[\mathrm{b}]}$, Daiane Mazza ${ }^{[c]}$, Giselly Marcato ${ }^{[\mathrm{d}]}$, \\ Monique Ribeiro ${ }^{[\mathrm{e}]}$, Thiago Absalão ${ }^{[\mathrm{f}]}$
}

[a] Mestre pela UFRJ, docente do curso de Fisioterapia da Universidade Estácio de Sá, Programa de Distúrbios da Coluna Vertebral, Laboratório de Biomecânica, Rio de Janeiro, RJ - Brasil, e-mail: professorfelipereis@yahoo.com.br

[b] Aluna do curso de Fisioterapia da Universidade Estácio de Sá, Programa de Distúrbios da Coluna Vertebral, Laboratório de Biomecânica, Rio de Janeiro, RJ - Brasil, e-mail: bruna.mafra@gmail.com

[c] Aluna do curso de Fisioterapia da Universidade Estácio de Sá, Programa de Distúrbios da Coluna Vertebral, Laboratório de Biomecânica, Rio de Janeiro, RJ - Brasil, e-mail: daianemazza@yahoo.com.br

[d] Aluna do curso de Fisioterapia da Universidade Estácio de Sá, Programa de Distúrbios da Coluna Vertebral, Laboratório de Biomecânica, Rio de Janeiro, RJ - Brasil, e-mail: giselly.marcato@hotmail.com

[e] Aluna do curso de Fisioterapia da Universidade Estácio de Sá, Programa de Distúrbios da Coluna Vertebral, Laboratório de Biomecânica, Rio de Janeiro, RJ - Brasil, e-mail: monique_949@hotmail.com

[f Aluna do curso de Fisioterapia da Universidade Estácio de Sá, Programa de Distúrbios da Coluna Vertebral, Laboratório de Biomecânica, Rio de Janeiro, RJ - Brasil, e-mail: thiagoabsalao@yahoo.com.br

\section{Resumo}

Introdução: A dor cervical mecânica é problema comum na população em geral e engloba a dor cervical aguda, as lesões em chicote, as disfunções cervicais e a dor cervical-ombro. A limitação da amplitude de movimento, a sensação de aumento da tensão muscular, a cefaleia, a braquialgia, a vertigem e outros sinais e sintomas são manifestações comuns e podem ser agravados por movimentos ou pela manutenção de posturas da coluna cervical. Estudos recentes mostram comprometimento no controle sensório-motor em pessoas com dor cervical manifestando-se por alterações da cinestesia cervical com dificuldade no reconhecimento da posição da cabeça, do movimento dos olhos e do equilíbrio. Objetivos: Descrever, com base na revisão da literatura, as manifestações e os métodos de avaliação dos distúrbios sensório-motores relacionados à dor cervical mecânica. Métodos: Para a revisão foram utilizadas as bases de dados de literatura científica indexada no período de 1965 a 2009. Considerou-se para a inclusão os artigos que abordassem a dor cervical mecânica e os distúrbios da propriocepção cervical, da coordenação dos movimentos dos olhos e do equilíbrio. Não houve restrição quanto à 
língua de publicação. O processo de seleção foi realizado por dois examinadores independentes, considerando a evidência científica em ordem decrescente, havendo preferência para as meta-análises e os estudos randomizados controlados. Resultados: Dos 119 artigos encontrados, 69 preenchiam os critérios de inclusão. Discussão: A presença de alterações dos músculos e das articulações cervicais, o processo de envelhecimento e a presença de dor cervical são descritos como fatores que alteram o sistema somatossensorial cervical e devem ser considerados também como perpetuantes. Conclusão: As alterações dos sistemas visual, do equilíbrio e proprioceptivo não podem ser desprezadas e devem ser consideradas durante a avaliação fisioterapêutica dos distúrbios cervicais, visto que existe uma integração entre os sistemas.

Palavras-chave: Cervicalgia. Cinestesia. Exame físico. Literatura de revisão.

\begin{abstract}
Introduction: Mechanical neck pain is a common health problem in general population including acute neck pain, wiplash injury, cervical dysfunction and neck-shoulder pain. Limitation in range of motion, muscular tension, headache, brachialgia, dizziness and others are the most common manifestations and can be aggravated by movements or maintained cervical posture. Recent studies describes alterations in cervical sensorymotor control showing cervical cinestesis disturbance with difficult in neck positioning, eye movement and balance. Objectives: Based in the literature review the aim of this study is to describe the manifestations and evaluation methods of the sensorymotor disturbs associated with mechanical neck pain. Methods: To this review, medical scientific papers published from 1965 to 2009 were used. As a including criteria it was considered studies about mechanical neck pain, cervical proprioception, eye movement coordination and balance. There were not restrictions in language. For the selection, two independents examers selected the studies according with the scientific evidence preferring metanalysis and randomized controlled trials. Results: From 119 studies 69 filled the including criteria and were selected. Discussion: The muscular and articular commitment of the cervical spine, the aging process, and mechanical neck pain were considered to be the most important factors to cause sensorymotor disturbance and should be considered as perpetuating factor. Conclusion: Alterations in visual, cervical proprioception and balance may be investigated in the physicaltherapist examination of all the patients with mechanical neck pain complaint.
\end{abstract}

Keywords: Neck pain. Kinesthesis. Physical examination. Review literature.

\title{
Introdução
}

A dor cervical mecânica é uma condição comum na sociedade moderna, que afeta a população de forma geral e que frequentemente torna-se crônica ou recorrente. Pode ser definida como distúrbios não específicos que comprometem esta região. Dentre as condições mais comuns enquadram-se as lesões em chicote (whiplash injury), o espasmo cervical, a síndrome cervical-ombro e os desarranjos mecânicos. São excluídas deste grupo todas as dores cervicais com diagnóstico, como hérnia de disco, a artrite reumatoide, doenças degenerativas, dentre outras (1).

Atualmente existe uma crescente correlação da dor cervical mecânica com alterações no sistema de controle postural. A principal modificação encontra-se na cinestesia cervical (reconhecimento da posição articular), no controle de movimento dos olhos e no equilíbrio estático (2-9).

O controle postural do corpo é o resultado da integração, pelo sistema nervoso central (SNC), de estímulos oriundos das aferências proprioceptivas do sistema somatossensorial cervical, do aparelho vestibular e da visão. Dessa maneira, qualquer comprometimento na integração dessas informações pode dar origem ao desequilíbrio corporal (10). 
Apesar de ser comum a presença de vertigens e alterações visuais nas pessoas com dor cervical, estas manifestações não são identificadas com frequência na prática clínica, ou quando estes sintomas são observados, acabam sendo atribuídos a doenças isoladas dos sistemas visual ou vestibular $(11,12)$.

Este estudo se baseia na revisão da literatura e tem como objetivo descrever as repercussões nos sistemas visual, do equilíbrio e proprioceptivo cervical provocadas pela dor cervical mecânica, destacando os métodos de avaliação.

\section{Metodologia}

Para o desenvolvimento do presente estudo, foram incluídos artigos indexados no período de 1965 a 2009. Utilizaram-se quatro bases de dados para constituição da revisão bibliográfica: Lilacs (Literatura LatinoAmericana e do Caribe em Ciências da Saúde), Medline (Literatura Internacional em Ciências da Saúde), Sciencedirect e SciELO. Os descritores utilizados para a busca dos artigos, de acordo com os descritores em ciências da saúde (DeCS), publicado pela Bireme, que é uma tradução do MeSH (Medical Subject Headings) da National Library of Medicine, foram: dor cervical mecânica, dor cervical e equilíbrio, dor cervical e propriocepção, dor cervical e avaliação, e suas transcrições para o inglês. Não houve restrições quanto à língua de publicação, sendo selecionados artigos que incluíssem revisões bibliográficas, tratamentos ou pesquisas experimentais. Dessa forma, dos 119 artigos encontrados, foram selecionados 69 artigos que tratassem do tema. Os demais excluídos estavam relacionados à abordagem clínica, eletroterapia, radiculopatia, orientações domicilares, técnicas de fisioterapia como massagem, cinesioterapia ou terapia manual que não abordavam os distúrbios somatossensoriais. Participaram da seleção dos artigos dois examinadores independentes que consideraram o tema principal para inclusão e o desenho do estudo. Após a seleção individual, foram discutidos e buscou-se um consenso quanto à seleção final.

\section{Controle sensório-motor cervical}

Os sistemas somatossensorial cervical (proprioceptivo), vestibular e visual recebem informações referentes à postura, posição e movimentos da cabeça e dos olhos respectivamente. Estes impulsos aferentes convergem em diversas regiões do córtex (12).

Para a integração entre os sistemas, foi demonstrado que o funcionamento adequado do complexo cabeça-pescoço-olhos está relacionado a informações provenientes dos receptores localizados na cápsula das articulações zigoapofisárias e aos músculos intrínsecos da região cervical (13). Estes estímulos trafegam pelas vias espino-vestibulares, que se dirigem aos núcleos vestibulares inferiores e ao vermis cerebelar, juntamente com as aferências oculares (14). Além disto, as informações proprioceptivas têm função de proteção das articulações, pela ativação dos mecanorreceptores e fusos neuromusculares, regulando o movimento articular $(15,16)$.

Os músculos localizados no espaço suboccipital apresentam grande número de fusos neuromusculares, o que contribui para a cinestesia cervical. Este controle envolve informações aferentes dos reflexos cérvico-coclear, cérvico-ocular e o tônico muscular cervical, que atuam em conjunto e são influenciados por informações provenientes do sistema vestibular e visual, permitindo o ajuste da posição da cabeça, dos olhos e da postura. O reflexo cérvico-coclear ativa os músculos da região cervical em resposta ao estiramento, com objetivo de manter a posição da cabeça (17). Já o cérvico-ocular atua em conjunto com o vestíbulo-ocular e o optocinético, agindo sobre os músculos extra-oculares para permitir a visão adequada durante o movimento (18). O tônico cervical está integrado ao vestíbulo-espinhal para controlar a postura (19).

As manifestações clínicas do comprometimento do sistema postural incluem nistagmo acompanhado de vertigem, otalgia, dor cervical, tensão muscular, podendo ou não estar associadas a zumbidos agudos e alteração auditiva. Estes sintomas mostram-se independentes dos níveis de ansiedade do paciente ou da medicação em uso, sendo explicados somente pelo distúrbio cervical (3, 20-23). 


\section{Integração da região cervical com o sistema visual e vestibular}

A integração dos três sistemas (proprioceptivo cervical, vestibular e óptico) é realizada principalmente pela presença de três reflexos: o cérvico-ocular, o vestíbulo-ocular e o optocinético. O reflexo cérvico-ocular age em conjunto com o reflexo vestíbulo-ocular e o optocinético para preservar a estabilidade da visão durante os movimentos da cabeça. Está definido que durante a rotação da cabeça estímulos aferentes proprioceptivos da musculatura cervical e das cápsulas articulares, principalmente de $\mathrm{C} 1$ a $\mathrm{C} 3$, alcançam o núcleo vestibular, permitindo que os olhos se movimentem para direção oposta em relação à cabeça (24). O reflexo vestíbuloocular pode ser subdividido em componentes rotacional e translacional, que estimulam os canais semicirculares e órgãos otolíticos, respectivamente. Quando a cabeça realiza a rotação, este reflexo permite que os olhos se movimentem em direção oposta (25). O reflexo optocinético é estimulado pelos movimentos captados pela visão e utiliza a velocidade relativa de deslocamento do objeto para mover os olhos na mesma direção. Assim como o cérvico-ocular, o reflexo optocinético responde predominantemente nos movimentos lentos da cabeça $(26,27)$.

Nos casos de disfunção cervical e comprometimento proprioceptivo, existe aumento da latência do reflexo vestíbulo-ocular e do cérvico-ocular, que se manifestam pela alteração no controle fino do movimento dos olhos. Isto foi observado nos momentos em que a cabeça permanecia na mesma posição, mas com o tronco em rotação de $45^{\circ}$. A hipótese que sustenta esta afirmação está pautada nas informações aferentes dos músculos cervicais (28). Considerando estes estudos, é esperado que pessoas com comprometimento cervical traumático ou insidioso apresentem déficits importantes no controle da postura e dos movimentos dos olhos $(5,29,30)$.

\section{Alterações proprioceptivas provocadas pela dor cervical mecânica}

O número de pessoas com disfunção cervical vem aumentando marcadamente nos últimos anos. A prevalência de dor cervical entre os adultos pode variar de $12-34 \%$ de toda a população com aproximadamente 10-19\% com queixa de dor persistente por mais de seis meses. Estima-se que 15\% das mulheres e 10\% dos homens tenham queixa de dor cervical em algum momento da vida. Este comprometimento, apesar de apresentar baixo índice de incapacidade, pode em alguns casos prejudicar as atividades diárias, influenciando negativamente a qualidade de vida (31-33).

As causas da dor cervical mecânica ainda não estão definidas. Alguns autores relatam não haver correlação com o grau de comprometimento articular e a dor $(34,35)$.

Em algumas pessoas que apresentam poucos sintomas ou dor cervical subclínica foram observados a diminuição da amplitude articular, resistência muscular e distúrbio da propriocepção. Além dessas queixas, ainda podem estar presentes a vertigem, perturbações visuais e alterações do equilíbrio (9). Como forma de prevenir a progressão destas alterações, é fundamental o diagnóstico das manifestações menores e abordagem adequada (36).

A principal modificação encontra-se na cinestesia cervical (reconhecimento da posição articular), no controle de movimento dos olhos e no equilíbrio estático (3, 4, 6-9, 24). Este comprometimento é observado tanto em pessoas com dor cervical traumática quanto nas insidiosas, seja aguda ou crônica (37-39). Em diversos estudos é relatada correlação entre dor cervical e o déficit proprioceptivo (2, 40-43).

O controle da postura da coluna cervical e do movimento depende de uma resposta motora adequada proveniente dos receptores articulares e dos fusos neuromusculares (44).

As hipóteses que relacionam distúrbios da coluna cervical com o desencadeamento de vertigem baseiam-se na potencialização das aferências sensitivas da região cervical, no comprometimento do sistema simpático cervical ou ainda na compressão vascular. Esta alteração da cinestesia cervical e a redução capacidade de permanecer com a cabeça em posição neutra podem contribuir para manutenção de posturas inadequadas, gerando sobrecarga para a coluna cervical e provocar dor (43).

Alguns mecanismos, sugeridos na literatura, provocam alterações no sistema somatossensorial cervical. $\mathrm{Na}$ ocorrência de trauma direto nos receptores musculares ou articulares, como nos casos das lesões por contragolpe conhecidas como lesões em chicote (whiplash injury) (2) - processos inflamatórios nos quais os mediadores inflamatórios podem ativar as terminações quimossensíveis e provocar uma resposta alterada 
dos fusos neuromusculares $(23,44)$-, os efeitos da dor nos nociceptores e mecanorreceptores prejudicam a modulação central aferente e, consequentemente, o controle neuromuscular da cervical (45-49).

\section{Avaliação dos distúrbios sensoriomotores na disfunção cervical}

De forma geral, a avaliação dos distúrbios sensoriomotores deve buscar identificar a presença de vertigem e as alterações da cinestesia cervical, do controle postural e da coordenação dos movimentos oculares. A descrição da vertigem é fundamental com relatos sobre os momentos em que surgem, os sintomas associados e a história de outros distúrbios. Outras queixas poderão estar presentes, como alterações visuais (opacificação, embaralhamento das letras, objetos com contornos mal definidos), do equilíbrio, história de quedas frequentes, dificuldade para locomover-se no escuro e necessidade de apoio para subir escadas. A avaliação objetiva da vertigem pode ser medida pelo Dizziness Handicap Inventory Short Form Questionnaire (50).

\section{Avaliação da posição articular cervical}

O teste de cinestesia cervical, que indica a capacidade movimentar a cabeça e retornar à posição inicial, tem sido usado com frequência na prática clínica e em pesquisas $(40,42,51,52)$.

Esta avaliação pode ser realizada fixando-se um laserpoint sobre a cabeça do paciente, que permanece sentado de frente para um alvo, a uma distância de $90 \mathrm{~cm}$. A posição inicial da cabeça deve ser marcada neste alvo e em seguida o paciente é solicitado a realizar os movimentos da coluna cervical com os olhos vendados e a retornar a posição de início. Verifica-se a precisão e acurácia com que o paciente consegue retornar à posição inicial. São considerados como apresentando alterações da cinestesia as pessoas cujos erros em relação à posição inicial forem maiores que $4 \mathrm{~cm}$ a $5 \mathrm{~cm}$ em qualquer direção (53).

\section{Avaliação do controle postural}

O controle postural é avaliado pela capacidade de manter o equilíbrio confortavelmente, por 30 segundos, com a diminuição da base de apoio para $10 \mathrm{~cm}$. Para este teste, o paciente permanece na posição ortostática com os olhos abertos; inicia-se a avaliação em uma superfície plana e rígida, e depois em uma superfície instável e flexível. Em seguida, repete-se o teste com os olhos fechados. Nos casos de pessoas com idade inferior a 45 anos é possível fazer o mesmo teste de forma unipodal. As alterações observadas são: a incapacidade de completar o teste, o aumento da oscilação e a rigidez na tentativa de manter o equilíbrio. Testes adicionais para se verificar o equilíbrio dinâmico e a marcha, o reflexo vestíbulo-ocular e a manobra de Hall-pike-Dix devem ser incluídos na suspeita do comprometimento vestibular (5, 9, 12).

\section{Avaliação dos movimentos dos olhos}

A coordenação dos movimentos oculares é testada pela (a) fixação dos olhos durante o movimento da cabeça, (b) fixação dos olhos em um ponto móvel enquanto a cabeça permanece fixa, (c) movimento rápido dos olhos acompanhando um ponto móvel, e (d) coordenação olhos/cabeça (12, 28).

\section{Teste de fixação dos olhos}

No teste de fixação dos olhos o paciente permanece sentado ou de pé e é solicitado a manter o olhar em um ponto fixo enquanto realiza os movimentos de flexão, extensão, rotação e inclinação da cabeça. 
A incapacidade de manter os olhos fixos, vertigem, diminuição da amplitude articular, opacidade da visão e náusea são sinais de uma resposta anormal (11).

\section{Teste dinâmico do olhar}

Este teste avalia a capacidade dos olhos de acompanhar um ponto que se move. Para isso, o paciente permanece sentado com a cabeça fixa, mantendo o foco em um ponto que é movido lentamente pelo examinador. O teste em seguida é realizado com a cabeça na mesma posição só com uma rotação de $45^{\circ}$ do tronco. Deve-se observar qualquer diferença no movimento dos olhos, comparado à posição inicial. Um achado frequente nas pessoas com dor cervical é a incapacidade de movimentar os olhos de forma coordenada - geralmente o acompanhamento perde-se e é seguido de um movimento rápido buscando novamente o foco. O teste mais avançado é executado com os mesmos posicionamentos,só que o objeto movendo-se com maior velocidade (54).

\section{Coordenação cabeça/olhos}

A coordenação dos olhos/cabeça é realizada solicitando ao paciente que primeiramente fixe o olhar em um ponto imóvel e em seguida movimente a cabeça mantendo o foco no ponto inicial $(10-12,55)$.

\section{Discussão}

O objetivo do presente estudo foi destacar a relação da dor cervical mecânica com alterações do sistema proprioceptivo cervical, dando ênfase às manifestações clínicas e à avaliação deste distúrbio. De acordo com esta revisão, a relação entre os sistemas visual, proprioceptivo e articular pode ser afetada por alguns fatores, como as lesões dos músculos suboccipitais profundos e das articulações relacionadas com os movimentos do pescoço, a presença de dor cervical e o envelhecimento podem influenciar, provocando alterações nos sistemas visual, relação e comprometendo o equilíbrio.

Alguns autores buscaram evidenciar a correlação dos músculos cervicais as alterações do equilíbrio. Ishikawa et al. (56) provocaram nistagmo, alteração do equilíbrio e ataxia severa ao realizar a secção dos nervos cervicais ou sua anestesia. Bove et al. (57) e Courtine et al. (58) relatam que, ao aplicar vibração para estimular os fusos neuromusculares da região cervical, induziram alterações importantes do equilíbrio, da posição dos olhos e da cabeça, além da dificuldade na marcha e corrida. Efeitos similares foram demonstrados por Schmid e Schieppati (59) e Vuillerme et al. (60), que com a contração isométrica provocavam a fadiga dos músculos da região cervical e reproduziram as alterações do equilíbrio. É possível que as alterações nos músculos cervicais provoquem impulsos aferentes anormais da região cervical para o sistema de controle postural (3, 12, 20-23).

Em um estudo de Lee, Wang e Wang (43), observou-se que indivíduos com episódios frequentes de dor cervical não foram capazes de posicionar a cabeça de forma precisa durante a avaliação. Este fato também foi relatado por Revel, Andre-Deshays e Minguet (40), Loudon, Ruhl e Field (41) e Feipel, Salvia, Klein e Rooze (42), e aponta evidências de uma relação entre o déficit proprioceptivo e a frequencia de dor cervical - ambos podem ser considerados como fatores perpetuantes entre si. Outros autores mostraram que a dor é capaz de induzir alterações nas descargas do fuso neuromuscular $(40,60)$ e nas propriedades proprioceptivas dos neurônios do tronco encefálico (2).

Além da dor cervical mecânica, é possível que o envelhecimento contribua para a alteração do controle postural cervical. Em um estudo de pessoas entre 18-66 anos, com história de lesão em chicote, foi observada a alteração da propriocepção nos indivíduos mais velhos (56). Entretanto, nos casos assintomáticos esta correspondência foi relatada somente para os movimentos no plano sagital, não estando presente nos demais (57). Teng et al. (61), em um estudo com indivíduos sem história de dor cervical, observou que a 
acurácia dos movimentos cervicais estava prejudicada nos adultos jovens. Este fato foi atribuído às alterações proprioceptivas que ocorrem nos receptores capsulares das articulações que sustentam carga, conforme estudado por outros autores (62-65). Foi postulado que qualquer alteração degenerativa nos discos intervertebrais ou nos músculos pode afetar a função dos receptores capsulares das facetas articulares ou vice-versa (66-69).

\section{Considerações finais}

De fato, o número de pessoas com dor cervical mecânica vem aumentando marcadamente nos últimos anos, em virtude de diversos fatores, como os acidentes automobilísticos e as funções exercidas durante atividades laborais. Considera-se que este distúrbio seja multifatorial não sendo possível estipular na maioria dos casos uma única causa, tendo fatores intrínsecos e/ou extrínsecos contribuindo na sua patogênese.

Além do comprometimento da coluna cervical, outras alterações ocorrem nos sistemas visual, proprioceptivo cervical e vestibular, manifestando-se por distúrbios visuais, no equilíbrio e vertigens.

Para a abordagem dos pacientes com dor cervical mecânica, é fundamental a avaliação adequada, tanto na coleta de informações referentes à história do paciente como no exame físico visando ao reconhecimento destas manifestações associadas. De acordo com a literatura abordada, é possível que as alterações destes sistemas dificultem a diminuição dos sintomas cervicais e que os programas que enfatizam o movimento coordenado dos olhos e a cinestesia cervical sejam eficazes, mesmo quanto utilizados isoladamente, reduzindo a administração de medicação, diminuindo a dor cervical e restaurando a noção de posição articular.

\section{Referências}

1. Hoving JL, Vet HC, Twisk JW, Deville WL, Koes BW, Bouter LM. Prognostic factors for neck pain in general practice. Pain. 2004;110(3):639-45.

2. Heikkila H, Astrom PG. Cervicocephalic kinesthetic sensibility in patients with whiplash injury. Scand J Rehabil Med. 1996;28(3):133-8.

3. Tjell C, Rosenhall U. Smooth pursuit neck torsion test: a specific test for cervical dizziness. Am J Otol. 1998;19(1):76-81.

4. Tjell C, Tenenbaum A, Sandstrom S. Smooth pursuit neck torsion test: a specific test for whiplash associated disorders? J Whiplash Relat Disord. 2002;1(2):9-24.

5. Treleaven J, Jull G, LowChoy N. Smooth pursuit neck torsion test in whiplash associated disorders-relationship to self reports of neck pain and disability, dizziness and anxiety. J Rehabil Med. 2005;37(4):219-23.

6. Kogler A, Lindfors J, Odkvist L, Ledin T. Postural stability using different neck positions in normal subjects and patients with neck trauma. Acta Otolaryngol. 2000;120(2):151-5.

7. Michaelson P, Michaelson M, Jaric S, Latash ML, Sjolander P, Djupsjobacka M. Vertical posture and head stability in patients with chronic neck. J Rehabil Med. 2003;35(5):229-35.

8. Sjöström H, Allum JH, Carpenter MG, Adkin AL, Honegger F, Ettlin T. Trunk sway measures of postural stability during clinical balance tests in patients with chronic whiplash injury symptoms. Spine. 2003;28(15):1725-34.

9. Treleaven J, Jull G, Sterling M. Dizziness and unsteadiness following whiplash injury: characteristic features and relationship with cervical joint position error. J Rehabil Med. 2003;35(1):36-43.

10. Greters ME, Bittar RSM, Bottino MA, Greters PM. Avaliação do tratamento fisioterápico na vertigem cervical (Estudo Preliminar). Arq Int Otorrinolaringol. 2007;11(4):406-10.

11. Treleaven J, Jull G, LowChoy N. The relationship of cervical joint position error to balance and eye movement disturbances in persistent whiplash. Man Ther 2006;11(2):99-106. 
12. Treleaven J. Sensorimotor disturbances in neck disorders affecting postural stability, head and eye movement control. Man Ther. 2008;13(1):2-11.

13. Cordo P, Gurfinkel VS, Bevan L, Kerr GK. Proprioceptive consequences of tendon vibration during movement. J Neurophysiol. 1995;74(4):1675-88.

14. Hulse M, Holzl M. Vestibulospinale reaktionen bei der zervikogenen Gleichgewiechtsstörung: die zervikogene Unsicherheit. HNO. 2000;48:295-301.

15. Johansson H, Djupsjobacka M, Sjolander P. Influences on the gamma muscle spindle system from muscle afferents stimulated by KCl and lactic acid. Neurosci Res. 1993;16(1):49-57.

16. Solomonow M, Zhou BH, Harris M, Lu Y, Baratta RV. The ligamento-muscular stabilizing system of the spine. Spine. 1998;23(32):2552-62.

17. Peterson BW. Current approaches and future directions to understanding control of head movement. Prog Brain Res. 2004;143:369-81.

18. Mergner T, Schweigart G, Botti F, Lehmann A. Eye movements evoked by proprioceptive stimulation along the body axis in humans. Exp Brain Res. 1998;120(4):450-60.

19. Yamagata Y, Yates BJ, Wilson VJ. Participation of Ia reciprocal inhibitory neurons in the spinal circuitry of the tonic neck reflex. Exp Brain Res. 1991;84(2):461-4.

20. Hildingsson C, Wenngren BI, Toolanen G. Eye motility dysfunction after soft-tissue injury of the cervical-spine: a controlled, prospective-study of 38 patients. Acta Orthop Scand. 1993;64(2):129-32.

21. Rubin AM, Woolley SM, Dailey VM, Goebel JA. Postural stability following mild head or whiplash injuries. Am J Otol. 1995;16(2):216-21.

22. Gimse R, Bjorgen IA, Tjell C, Tyssedal JS, Bo K. Reduced cognitive functions in a group of whiplash patients with demonstrated disturbances in the posture control system. J Clin Exp Neuropsychol 1997;19(6):838-49.

23. Wenngren B, Pettersson K, Lowenhielm G, Hildingsson C. Eye motiliy and auditory brainstem response dysfunction after whiplash injury. Acta Orthop Scand 2002;122(3):276-83.

24. Hikosaka O, Maeda M. Cervical effects on abducens motoneurons and their interaction with vestibulo-ocular reflex. Exp Brain Res. 1973;18(5):512-30.

25. Tabak S, Collewijn H, Boumans LJ, van der Steen J. Gain and delay of human vestibulo-ocular reflexes to oscillation and steps of the head by a reactive torque helmet, I: normal subjects. Acta Otolaryngol. 1997;117(6):785-95.

26. Mergner T, Schweigart G, Botti F, Lehmann A. Eye movements evoked by proprioceptive stimulation along the body axis in humans. Exp Brain Res. 1998;120(4):450-60.

27. Kelders WP, Kleinrensink GJ, van der Geest JN, Feenstra L, de Zeeuw CI, Frens MA. Compensatory increase of the cervico-ocular reflex with age in healthy humans. J Physiol. 2003;553(Pt 1):311-7.

28. Montfoort I, Kelders WP, van der Geest JN, Schipper IB, Feenstra L, de Zeeuw CI, et al. Interaction between ocular stabilization reflexes in patients with whiplash injury. Invest Ophthalmol Vis Sci. 2006;47(7):2881-4.

29. Storaci R, Manelli A, Schiavone N, Mangia L, Prigione G, Sangiorgi S. Whiplash injury and oculomotor dysfunctions: clinical-posturographic correlations. Europ Spine J 2006;15(12):1811-6.

30. Field S, Treleaven J, Jull G. Standing balance: a comparison between idiopathic and whiplash induced neck pain. Man Ther. 2008;13(3):183-91.

31. Bovim G, Schrader H, Sand T. Neck pain in the general population. Spine. 1994;19(12):1307-9.

32. Dvorak J. Epidemiology, physical examination, and neurodiagnostics. Spine. 1998;23(24):2663-73. 
33. Cote P, Cassidy JD, Carroll L. The factors associated with neck pain and its related disability in the Saskatchewan population. Spine. 2000;25(9):1109-17.

34. Marchiori DM, Henderson CN. A cross-sectional study correlating cervical radiographic degenerative findings to pain and disability. Spine. 1996;21(23):2747-51.

35. Harrison DE, Bula JM, Gore DR. Roentgenographic findings in the cervical spine in asymptomatic persons: a 10-year follow-up. Spine. 2002;27(11):1249-50.

36. Lee H, Nicholson LL, Adams RD, Bae SS. Proprioception and rotation range sensitization associated with subclinical neck pain. Spine. 2005;30(3):E60-7.

37. Rix G, Bagust J. Cervicocephalic kinesthetic sensibility in patients with chronic, nontraumatic cervical spine pain. Arch Phys Med Rehabil. 2001;82(7):911-9.

38. Armstrong BS, McNair PJ, Williams M. Head and neck position sense in whiplash patients and healthy individuals and the effect of the cranio-cervical flexion action. Clin Biomech. 2005;20(7):675-84.

39. Zito G, Jull G, Story I. Clinical tests of musculoskeletal dysfunction in the diagnosis of cervicogenic headache. Man Ther. 2006;11(2):118-29.

40. Revel M, Andre-Deshays C, Minguet M. Cervicocephalic kinesthetic sensibility in patients with cervical pain. Arch Phys Med Rehabil. 1991;72(5):288-91.

41. Loudon JK, Ruhl M, Field E. Ability to reproduce head position after whiplash injury. Spine. 1997;22(8):865-8.

42. Feipel V, Salvia P, Klein H, Rooze M. Head repositioning accuracy in patients with whiplash-associated disorders. Spine. 2006;31(2):E51-8.

43. Lee HY, Wang JD, Yao G, Wang SF. Association between cervicocephalic kinesthetic sensibility and frequency of subclinical neck pain. Man Ther. 2008;13(5):419-25.

44. Kristjansson E. The cervical spine and proprioception. In: Boyling J, Jull G, editors. Grieve's modern manual therapy: the vertebral column. 3rd ed. Edinburgh: Churchill Livingstone; 2005. p. 243-56.

45. de Jong PT, de Jong JM, Cohen B, Jongkees LB. Ataxia and nystagmus induced by injection of local anesthetics in the neck. Ann Neurol. 1977;1(3):240-6.

46. Thunberg J, Hellström F, Sjölander P, Bergenheim M, Wenngren B, Johansson H. Influences on the fusimotormuscle spindle system from chemosensitive nerve endings in the cervical facet joints in the cat; possible implications for whiplash induced disorders. Pain. 2001;91(12):15-22.

47. Le Pera D, Graven-Nielsen T, Valeriani M, Oliviero A, Di Lazzaro V, Tonali PA, et al. Inhibition of motor system excitability at cortical and spinal level by tonic muscle pain. Clin Neurophysiol. 2001;112(9):1633-41.

48. Wenngren BI, Pedersen J, Sjölander P, Bergenheim M, Johansson H. Bradykinin and muscle stretch alter contralateral cat neck muscle spindle output. Neurosci Res. 1998;32(2):119-29.

49. Ageborg E. Consequences of a ligament injury on neuromuscular function and relevance to rehabilitation: using the anterior cruciate ligament-injured knee as a model. J Electromyogr Kinesiol. 2002;12(3):205-12.

50. Tesio L, Alpini D, Cesarani A, Perucca L. Short form of the dizziness handicap inventory. Am J Phys Med Rehabil. 1999;78(3):233-41.

51. Sterling M, Jull G, Vicenzino B, Kenardy J, Darnell R. Development of motor system dysfunction following whiplash injury. Pain. 2003;103(12):65-73.

52. Treleaven J, Jull G, Lowchoy N. The relationship of cervical joint position error to balance and eye movement disturbances in persistent whiplash. Man Ther. 2005;11(2):99-106. 
53. Revel M, Minguet M, Gregoy P, Vaillant J, Manuel JL. Changes in cervicocephalic kinesthesia after a proprioceptive rehabilitation program in patients with neck pain: a randomized controlled study. Arch Phys Med Rehabil. 1994;75(8):895-9.

54. Prushansky T, Dvir Z, Pevzner E, Gordon CR. Electro-oculographic measures in patients with chronic whiplash and healthy subjects: a comparative study. J Neurol Neurosurg Psychiatry. 2004;75(11):1642-4.

55. Edmondston SJ, Chan HY, Ngai GC, Warren ML, Williams JM, Glennon S, et al. Postural neck pain: an investigation of habitual sitting posture, perception of 'good' posture and cervicothoracic kinaesthesia. Man Ther. 2007;12(4):363-71.

56. Ishikawa K, Matsuzaki Z, Yokomizo M, Terada N, Miyazaki S, Togawa K. Effect of unilateral section of cervical afferent nerve upon optokinetic response and vestibular nystagmus induced by sinusoidal rotation in guinea pigs. Acta Otolaryngol Suppl. 1998;537:6-10.

57. Bove M, Courtine G, Schieppati M. Neck muscle vibration and spatial orientation during stepping in place in humans. J Neurophysiol. 2002;88(5):2232-41.

58. Courtine G, Papaxanthis C, Laroche D, Pozzo T. Gait-dependent integration of neck muscle afferent input. Neuroreport. 2003;14(18):2365-8.

59. Schmid M, Schieppati M. Neck muscle fatigue and spatial orientation during stepping in place in humans. J Appl Physiol. 2005;99(1):141-53.

60. Vuillerme N, Pinsault N, Vaillant J. Postural control during quiet standing following cervical muscular fatigue: effects of changes in sensory inputs. Neurosci Lett. 2005;378(3):135-9.

61. Teng CC, Chai H, Lai DM, Wang SF. Cervicocephalic kinesthetic sensibility in young and middle-aged adults with or without a history of mild neck pain. Man Ther. 2007;12(1):22-8.

62. Mense S, Skeppar P. Discharge behavior of feline gamma-motoneurones following induction of an artificial myositis. Pain. 1991;46(2):201-10.

63. Johansson H, Djupsjobacka M, Sjolander P. Influences on the gammamuscle spindle system from muscle afferents stimulated by $\mathrm{KCl}$ and lactic acid. Neurosci Res. 1993;16(1):49-57.

64. Thunberg J, Ljubisavljevic M, Djupsjobacka M, Johansson H. Effects on the fusimotor-muscle spindle system induced by intramuscular injections of hypertonic saline. Exp Brain Res. 2002;142(3):319-26.

65. Capra NF, Ro JY. Experimental muscle pain produces central modulation of proprioceptive signals arising from jaw muscle spindles. Pain. 2000;86(12):151-62.

66. Skinner HB, Barrack RL, Cook SD. Age-related decline in proprioception. Clin Orthop Relat Res. 1984;(184):208-11.

67. Gilsing MG, Van den Bosch CG, Lee SG, Ashton-Miller JA, Alexander NB, Schultz AB, et al. Association of age with the threshold for detecting ankle inversion and eversion in upright stance. Age Ageing. 1995;24(1):58-66.

68. Robbins S, Waked E, McClaran J. Proprioception and stability: foot position awareness as a function of age and footwear. Age Ageing. 1995;24(1):67-72.

69. Pai YC, Rymer WZ, Chang RW, Sharma L. Effect of age andosteoarthritis on knee proprioception. Arthritis Rheuma. 1997;40(12):2260-5.

Recebido: 09/06/2008

Received: 06/09/2008

Aprovado: 27/07/2010

Approved: 07/27/2010 